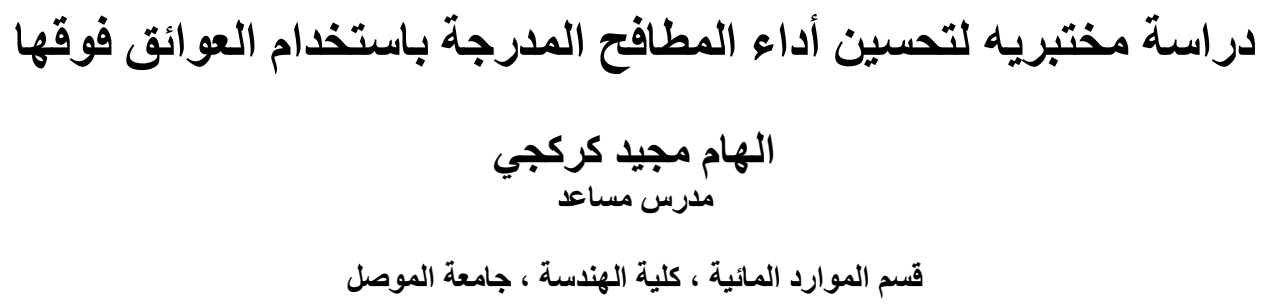

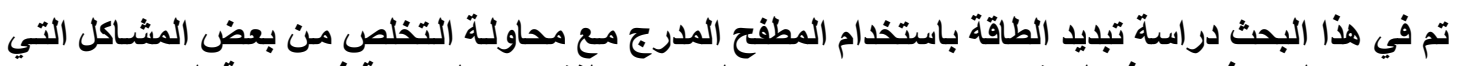

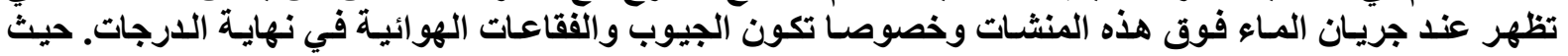

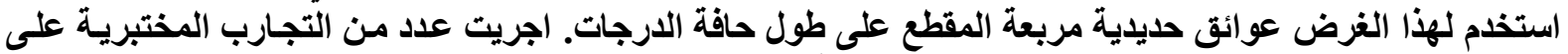

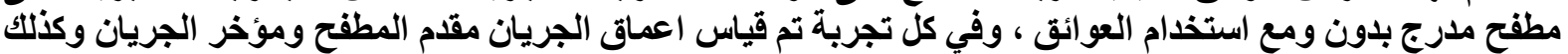

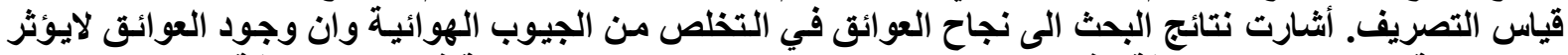

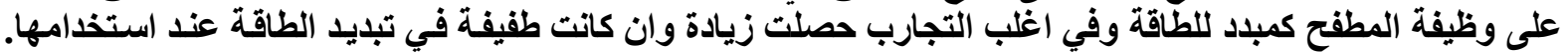

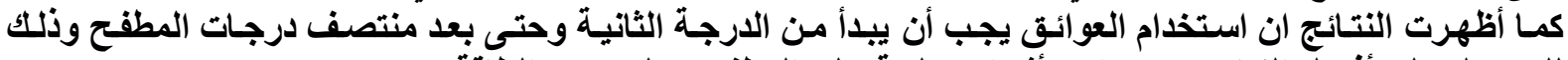

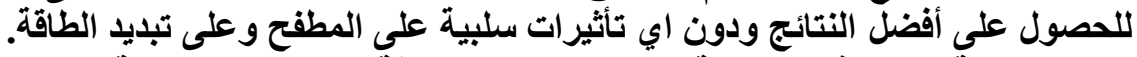

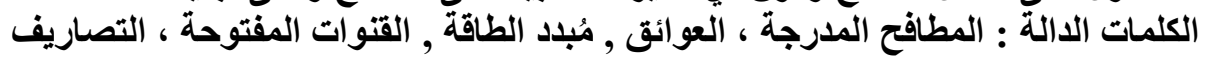

\title{
Experimental Study for Improving the performance of Stepped Spillway using an Obstructions
}

\author{
Elham Majeed Kurukji \\ Water Resources Dept., College of Eng., Mosul Univ.
}

\begin{abstract}
In this paper the energy dissipation was studied using stepped spillway, in addition to that the elimination of air pockets which take place at steps was also studied by using obstructions along the edge of steps. These obstructions were made from iron with square cross-section. Several experiments were conducted on stepped spillway with and without obstructions. The results of the experiments showed that these obstructions were very successful in eliminating air pockets. Their present in general had a positive effect on energy dissipation along the stepped spillway. The results also indicated that the use of these obstructions should be started from second step until the middle step of the spillway.
\end{abstract}

Keywords: stepped spillway, obstructions, energy dissipater, open channels, discharges. 


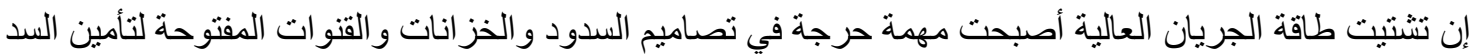

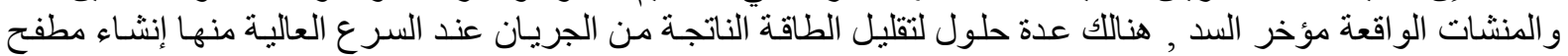

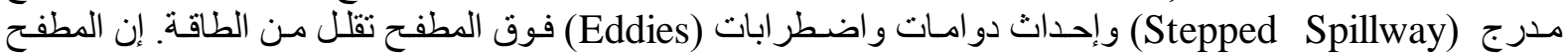

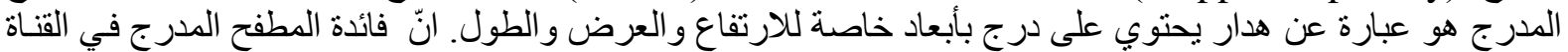

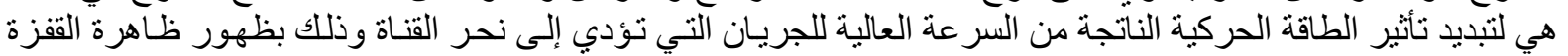

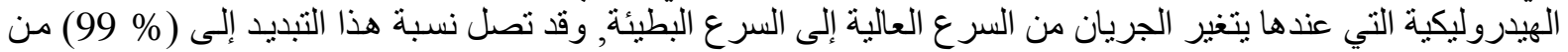

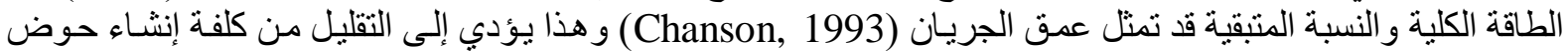

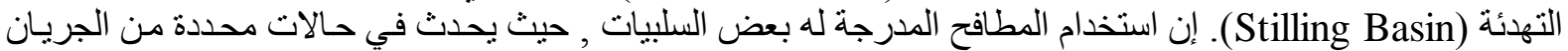

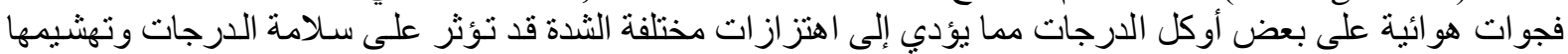

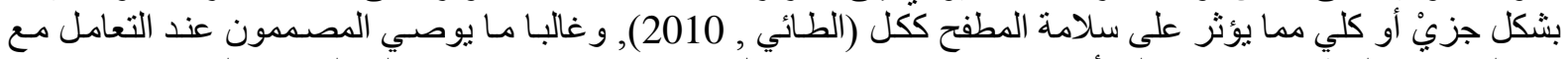

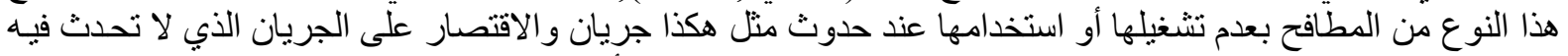

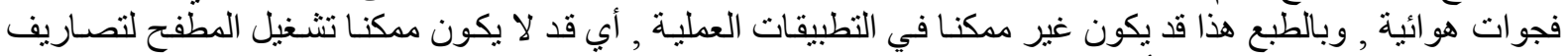

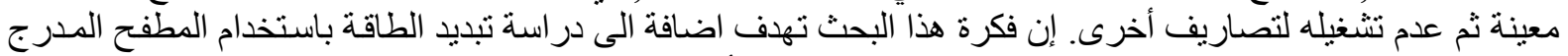

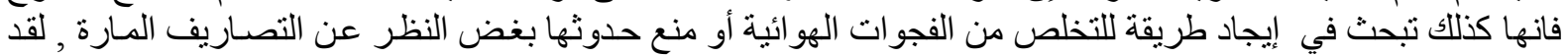

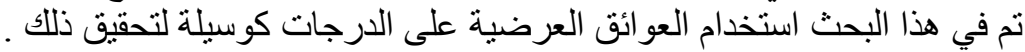

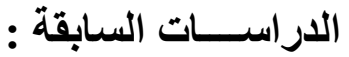

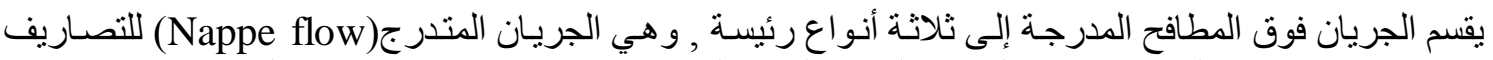

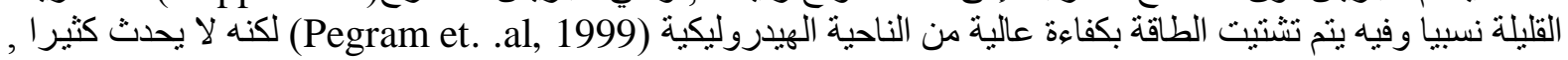

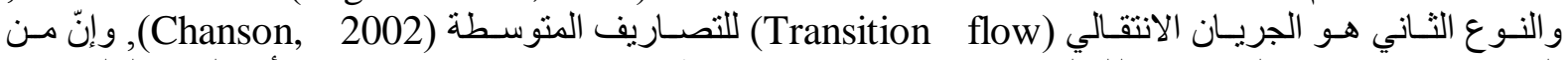

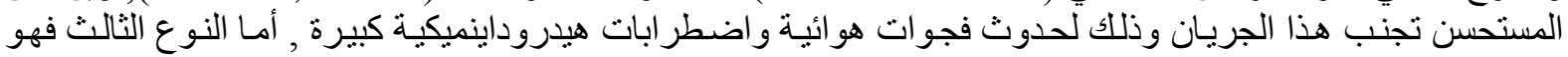

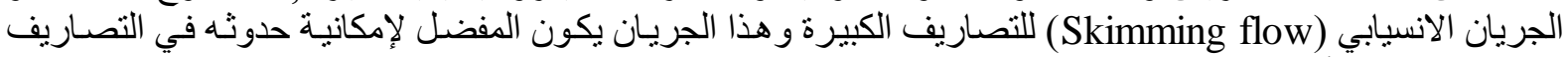

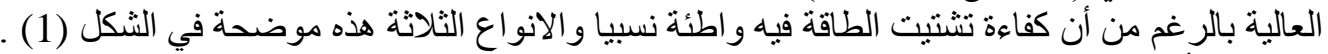
لقد أجريت عدة در اسات من قبل الباحثين

(Sorenson, 1985), (Peyras 1992 ) , (Chanson, 1994), (Christodoulou, 1995), (Ahman and Zapel, 2000)،( Peruginelli and Pagliara, 2000).

و استتنجو ا أن تشتيت الطاقة فوق المطافح المدرجة يعتمد على التصـاريف وانحدار المطفح , إضـافة إلى الثكل الهندسي

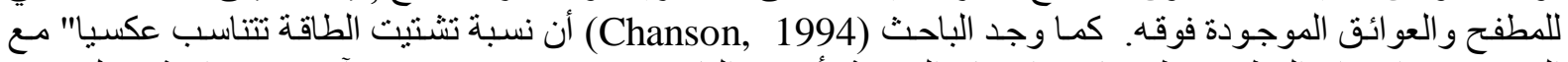

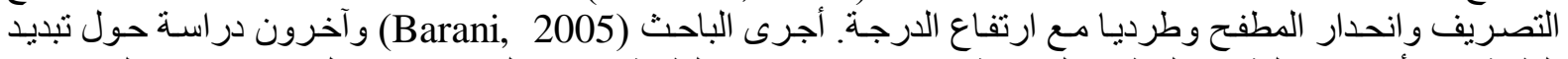

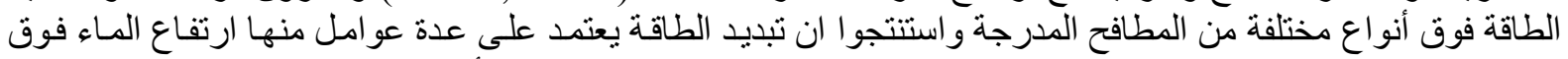

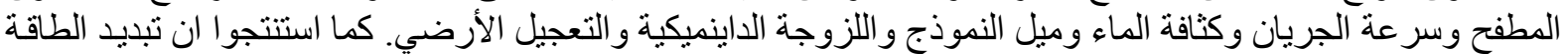

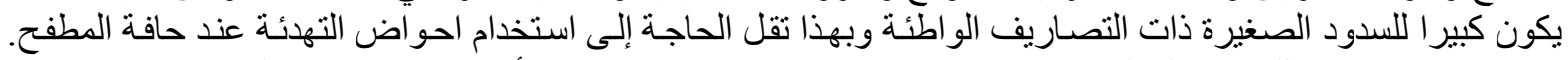

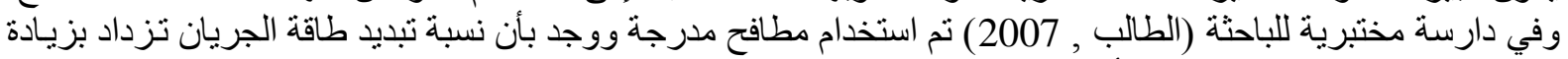

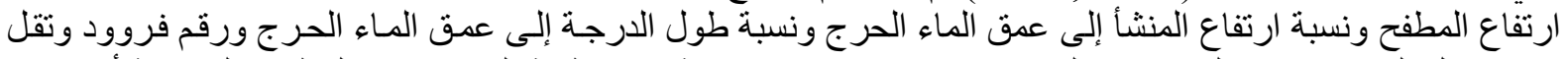

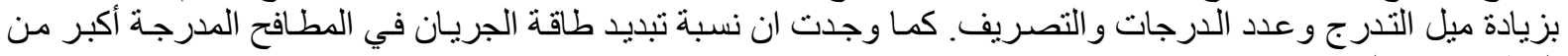
المطافح غير المدرجة.

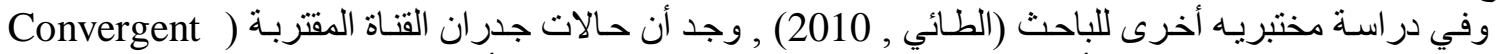

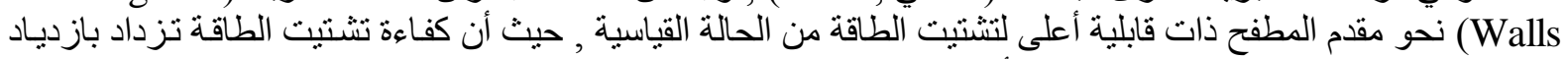

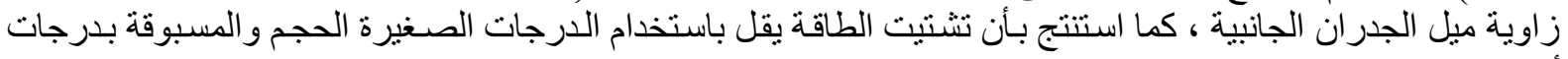




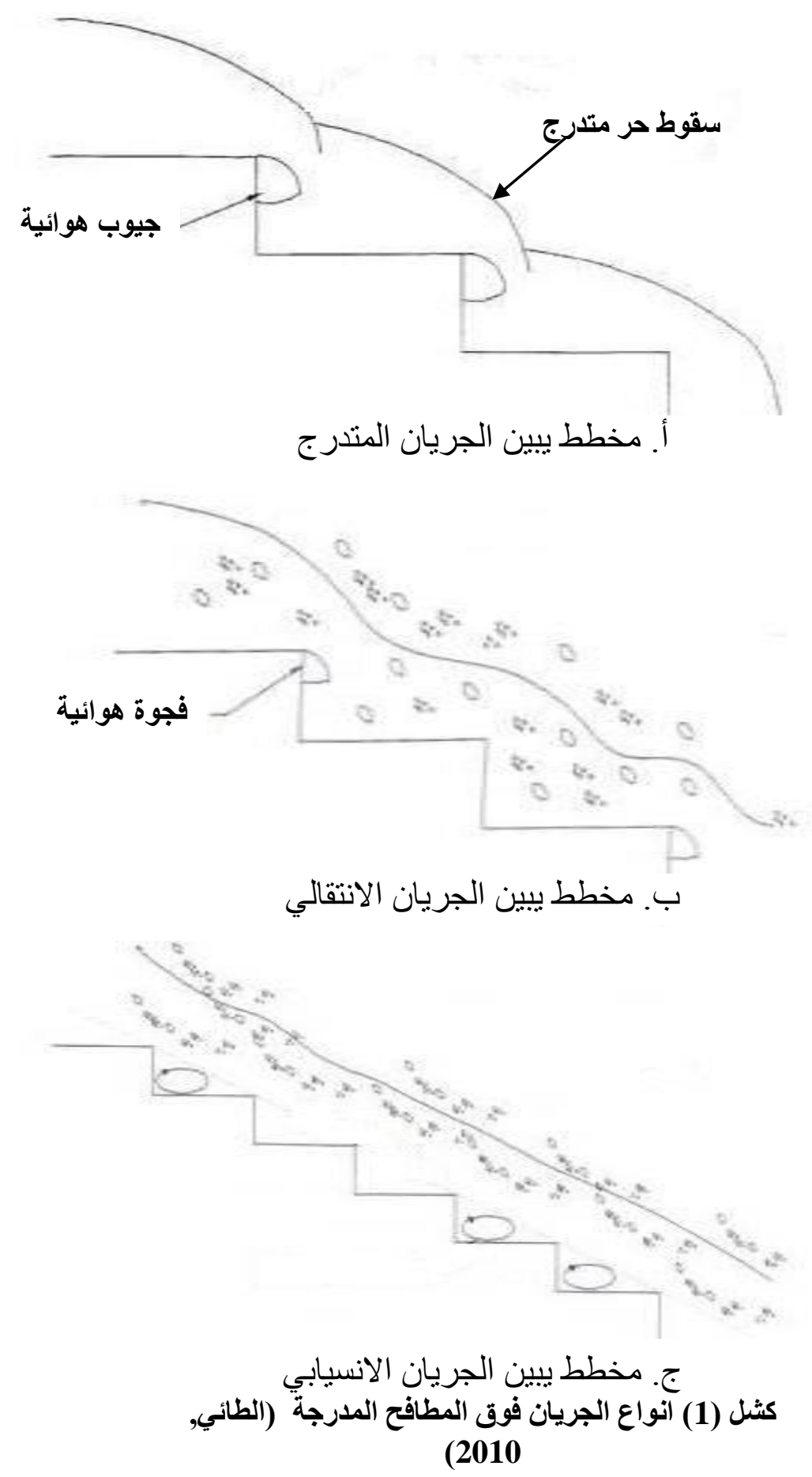

العمل ألمختبري:

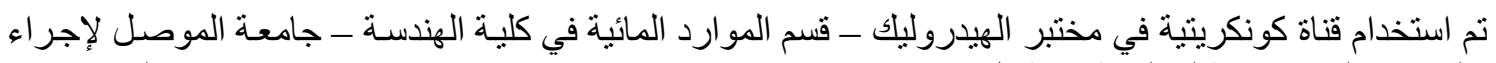

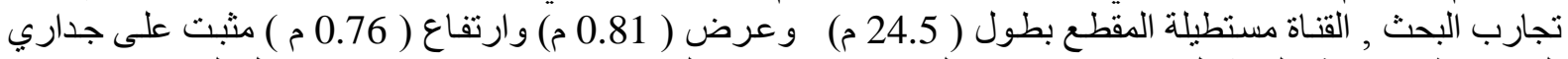

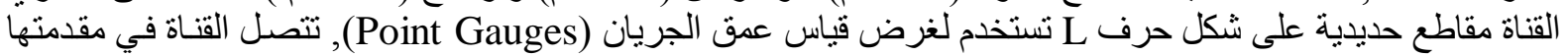
بحوض تهيئة للجريـان قبل دخوله إلى القناة وفي نهايـة القناة حوض تصريف بطول ( 8 م ) و وعرض (P) (0.8 م) وارتفاع 


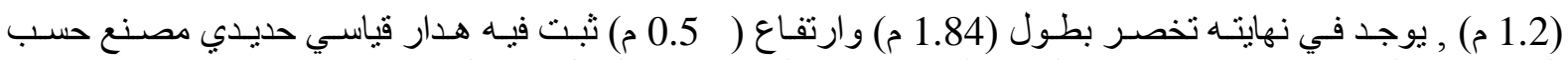

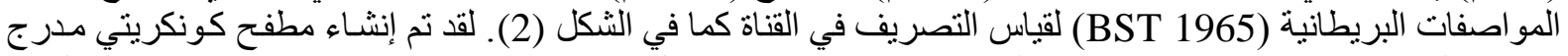

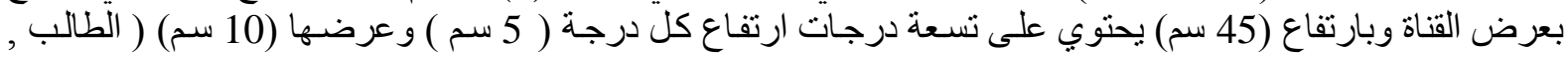

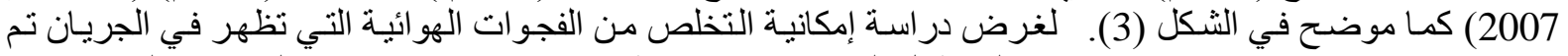

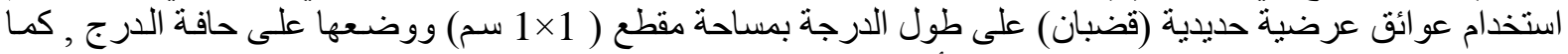

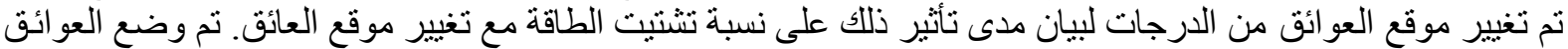

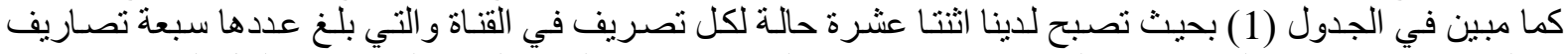

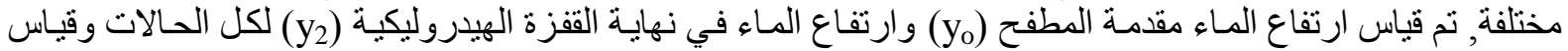
منسوب الماء فوق الهدار القياسي (H) وباء

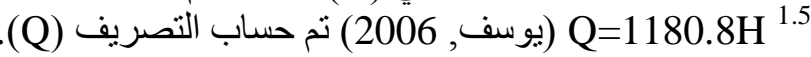
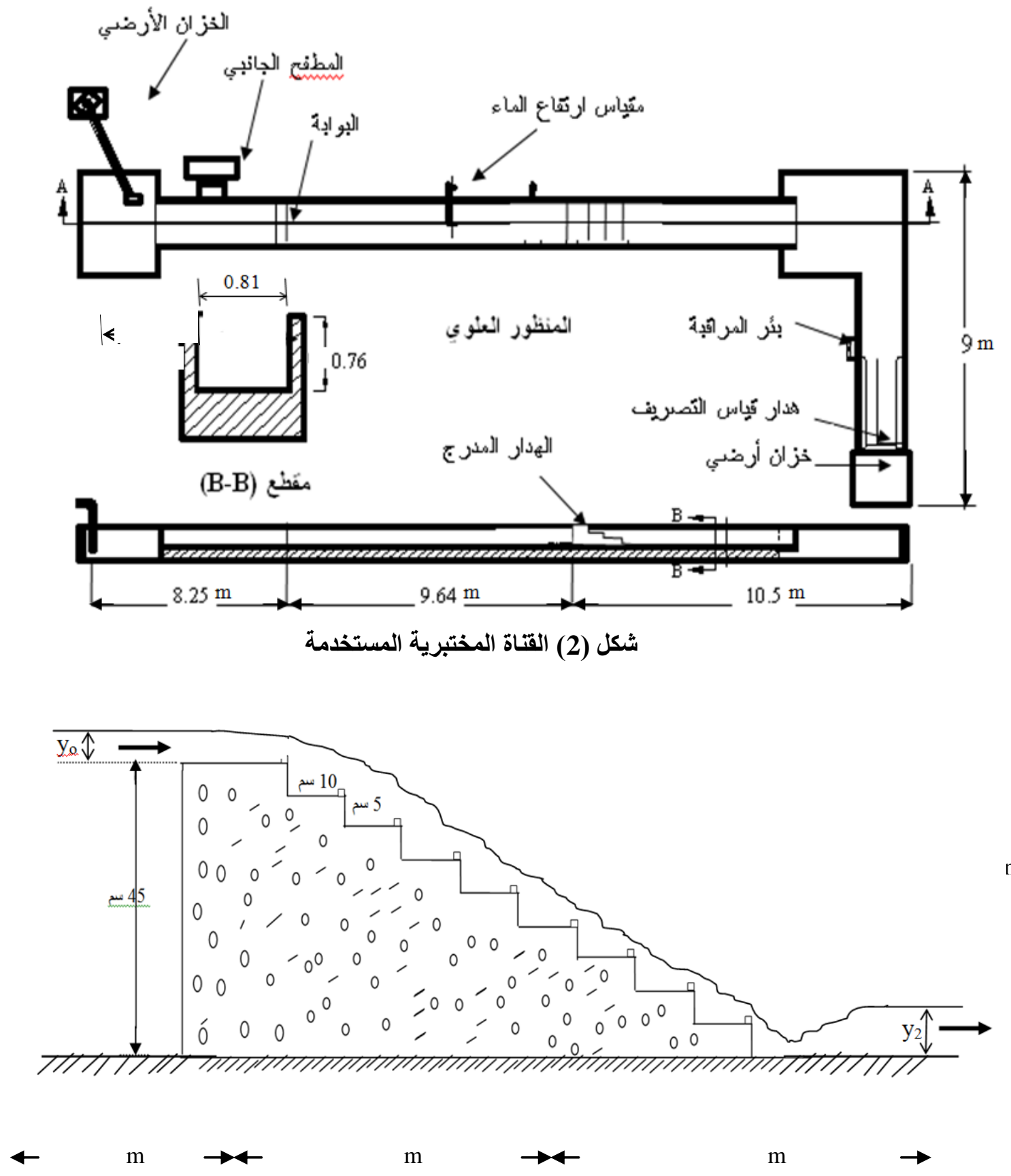

شكل (3) مقطع في المطقح الخرساني المستخدم 


\section{جدول (1): يبين الحالات التي استخدمت فيها العوائق}

\begin{tabular}{|c|c|c|c|c|c|c|c|c|c|}
\hline \multicolumn{10}{|c|}{ رقم الدرجـــة ابتداء من الأعلى } \\
\hline 9 & 8 & 7 & 6 & 5 & 4 & 3 & 2 & 1 & الحـالة \\
\hline & & & & & & & & & الأولى \\
\hline & & & & & & & & - & الثانية \\
\hline & & & & & & & - & - & الثالثة \\
\hline & & & & & & - & - & - & الر ابعة \\
\hline & & & & & - & - & - & - & الخامسة \\
\hline & & & & - & - & - & - & - & السادسة \\
\hline & & & - & - & - & - & - & - & السابعة \\
\hline & & - & - & - & - & - & - & - & الثامنة \\
\hline & - & - & - & - & - & - & - & - & التاسعة \\
\hline - & - & - & - & - & - & - & - & - & العاثرة \\
\hline & & & & & & - & & & الحادية عشر \\
\hline & & - & & & & & & & الثانية عشر \\
\hline
\end{tabular}

الإشارة (_-) تعني استخدام العوائق عند الدرجة المحددة.

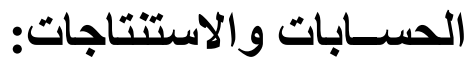

لمعرفة نسبة تشتيت طاقة الجريان باستخدام المطفح الهيدروليكي المدرج نم إجراء الحسابات التالية :

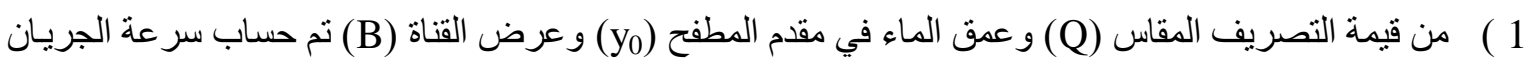
مقدم المطفح (Vo) من معادلة الاستمر ارية:

$$
\begin{aligned}
\mathrm{Q} & =\mathrm{V}_{\mathrm{o}}^{*} \mathrm{y}_{\mathrm{o}}^{* \mathrm{~B}} \\
\mathrm{E}_{\mathrm{t}} & =\mathrm{P}+\left(\mathrm{V}_{\mathrm{o}}^{2} / 2 * \mathrm{~g}\right)+\mathrm{y}_{\mathrm{o}}
\end{aligned}
$$

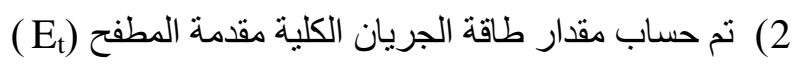

$$
\text { حيث ان : }
$$

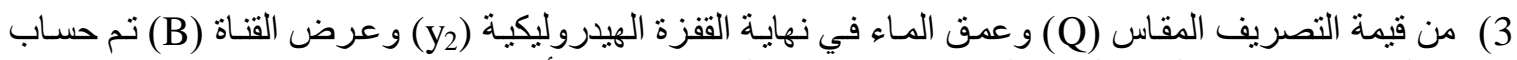
$\mathrm{Q}=\mathrm{V}_{2} * \mathrm{y}_{2} * \mathrm{~B}$ سرعة الجريان في نهاية القفزة الهيدروليكية (V) من معادلة الاستمر ارية أيضا:

$$
\begin{aligned}
& \mathrm{Fr}_{2}=\mathrm{V}_{2} /\left(\mathrm{g}^{*} \mathrm{y}_{2}\right){ }^{0.5} \\
& \text { 4) من ذلك تم حساب قيمة رقم فرود بعد القفزة الهيدروليكية (Fr2) } \\
& \mathrm{E}_{2}=\mathrm{y}_{2}+\left(\mathrm{V}_{2}^{2} / 2 \mathrm{~g}\right) \\
& \text { 5) حساب مقدار طاقة الجريان عند مؤخر الققزة الهيدروليكية (E2) باستخدام المعادلة: } \\
& \text { 6) نسبة تبديد طاقة الجريان بين مقدم ومؤخر المطفح: }
\end{aligned}
$$

$$
\triangle \mathrm{E} \%=\left(\left(\mathrm{E}_{\mathrm{t}}-\mathrm{E}_{2}\right) / \mathrm{E}_{\mathrm{t}}\right) * 100
$$


جدول (2): يبين الحسابات والنتائج

\begin{tabular}{|c|c|c|c|c|c|c|c|c|c|c|c|}
\hline$\underline{E}$ & $\underline{E}$ & $\begin{array}{r}\mathbf{y}_{\mathbf{o}} \\
\mathrm{cm}\end{array}$ & $\begin{array}{c}\mathbf{Q} \\
\mathrm{Cm}^{3} / \mathrm{sec}\end{array}$ & $\begin{array}{c}\mathbf{y}_{\mathbf{2}} \\
\mathrm{cm}\end{array}$ & $\begin{array}{c}\mathbf{V}_{\mathbf{o}} \\
\mathrm{Cm} / \mathrm{sec}\end{array}$ & $\begin{array}{l}\mathbf{E}_{\mathrm{t}} \\
\mathrm{cm}\end{array}$ & $\begin{array}{c}\mathbf{V}_{2} \\
\mathrm{Cm} / \mathrm{sec}\end{array}$ & $\mathbf{F r}$ & $\begin{array}{r}\mathbf{E}_{2} \\
\mathrm{~cm}\end{array}$ & $\Delta \mathrm{E} \%$ & ألنواع \\
\hline 1 & 1 & 2.2 & 1956 & 7.4 & 10.98 & 47.2 & 3.26 & 0.0383 & 7.41 & 84.31 & \multirow{12}{*}{$\stackrel{\overbrace{}}{\tilde{E}}$} \\
\hline & 2 & 3.7 & 1956 & 6.5 & 6.53 & 48.7 & 3.72 & 0.0465 & 6.51 & 86.64 & \\
\hline & 3 & 3.7 & 1956 & 7 & 6.53 & 48.7 & 3.45 & 0.0416 & 7.01 & 85.61 & \\
\hline & 4 & 3.7 & 1956 & 6.9 & 6.53 & 48.7 & 3.50 & 0.0425 & 6.91 & 85.82 & \\
\hline & 5 & 3.7 & 1956 & 6.9 & 6.53 & 48.7 & 3.50 & 0.0425 & 6.91 & 85.82 & \\
\hline & 6 & 3.7 & 1956 & 6.8 & 6.53 & 48.7 & 3.55 & 0.0435 & 6.81 & 86.02 & \\
\hline & 7 & 3.7 & 1956 & 6.8 & 6.53 & 48.7 & 3.55 & 0.0435 & 6.81 & 86.02 & \\
\hline & 8 & 3.7 & 1956 & 6.7 & 6.53 & 48.7 & 3.60 & 0.0445 & 6.71 & 86.23 & \\
\hline & 9 & 3.7 & 1956 & 6.7 & 6.53 & 48.7 & 3.60 & 0.0445 & 6.71 & 86.23 & \\
\hline & 10 & 3.7 & 1956 & 6.8 & 6.53 & 48.7 & 3.55 & 0.0435 & 6.81 & 86.02 & \\
\hline & 11 & 2 & 1956 & 6.7 & 12.07 & 47 & 3.60 & 0.0445 & 6.71 & 85.73 & \\
\hline & 12 & 2 & 1956 & 6.9 & 12.07 & 47 & 3.50 & 0.0425 & 6.91 & 85.31 & \\
\hline \multirow[t]{12}{*}{2} & 1 & 4.2 & 3853 & 8.8 & 11.33 & 49.2 & 5.41 & 0.0582 & 8.81 & 82.08 & \multirow{24}{*}{ E } \\
\hline & 2 & 5 & 3853 & 8.6 & 9.51 & 50 & 5.53 & 0.0602 & 8.62 & 82.77 & \\
\hline & 3 & 5 & 3853 & 8.45 & 9.51 & 50 & 5.63 & 0.0618 & 8.47 & 83.07 & \\
\hline & 4 & 5 & 3853 & 8.2 & 9.51 & 50 & 5.80 & 0.0647 & 8.22 & 83.57 & \\
\hline & 5 & 5 & 3853 & 8.2 & 9.51 & 50 & 5.80 & 0.0647 & 8.22 & 83.57 & \\
\hline & 6 & 5.1 & 3853 & 8.4 & 9.33 & 50.1 & 5.66 & 0.0624 & 8.42 & 83.20 & \\
\hline & 7 & 5.1 & 3853 & 8.3 & 9.33 & 50.1 & 5.73 & 0.0635 & 8.32 & 83.40 & \\
\hline & 8 & 5.1 & 3853 & 8.5 & 9.33 & 50.1 & 5.60 & 0.0613 & 8.52 & 83.00 & \\
\hline & 9 & 5.1 & 3853 & 8.3 & 9.33 & 50.1 & 5.73 & 0.0635 & 8.32 & 83.40 & \\
\hline & 10 & 5.1 & 3853 & 8.5 & 9.33 & 50.1 & 5.60 & 0.0613 & 8.52 & 83.00 & \\
\hline & 11 & 3.7 & 3853 & 8.3 & 12.86 & 48.7 & 5.73 & 0.0635 & 8.32 & 82.92 & \\
\hline & 12 & 3.6 & 3853 & 8.6 & 13.21 & 48.6 & 5.53 & 0.0602 & 8.62 & 82.27 & \\
\hline \multirow[t]{12}{*}{3} & 1 & 4.6 & 10164 & 9.6 & 27.28 & 49.6 & 13.07 & 0.1347 & 9.69 & 80.47 & \\
\hline & 2 & 6 & 10164 & 9 & 20.91 & 51 & 13.94 & 0.1484 & 9.10 & 82.16 & \\
\hline & 3 & 6 & 10164 & 8.8 & 20.91 & 51 & 14.26 & 0.1535 & 8.90 & 82.54 & \\
\hline & 4 & 6 & 10164 & 8.9 & 20.91 & 51 & 14.10 & 0.1509 & 9.00 & 82.35 & \\
\hline & 5 & 6 & 10164 & 9.1 & 20.91 & 51 & 13.79 & 0.1459 & 9.20 & 81.97 & \\
\hline & 6 & 6 & 10164 & 9.3 & 20.91 & 51 & 13.49 & 0.1413 & 9.39 & 81.58 & \\
\hline & 7 & 6 & 10164 & 9.35 & 20.91 & 51 & 13.42 & 0.1401 & 9.44 & 81.49 & \\
\hline & 8 & 6 & 10164 & 9.2 & 20.91 & 51 & 13.64 & 0.1436 & 9.29 & 81.77 & \\
\hline & 9 & 6 & 10164 & 9.2 & 20.91 & 51 & 13.64 & 0.1436 & 9.29 & 81.77 & \\
\hline & 10 & 6 & 10164 & 9.2 & 20.91 & 51 & 13.64 & 0.1436 & 9.29 & 81.77 & \\
\hline & 11 & 4.6 & 10164 & 9.5 & 27.28 & 49.6 & 13.21 & 0.1368 & 9.59 & 80.67 & \\
\hline & 12 & 4.6 & 10164 & 9.5 & 27.28 & 49.6 & 13.21 & 0.1368 & 9.59 & 80.67 & \\
\hline \multirow[t]{4}{*}{4} & 1 & 6.05 & 18229 & 10.6 & 37.20 & 51.05 & 21.23 & 0.2082 & 10.83 & 78.79 & \multirow{4}{*}{ E. } \\
\hline & 2 & 7.3 & 18229 & 10.7 & 30.83 & 52.3 & 21.03 & 0.2053 & 10.93 & 79.11 & \\
\hline & 3 & 7.3 & 18229 & 10.2 & 30.83 & 52.3 & 22.06 & 0.2206 & 10.45 & 80.02 & \\
\hline & 4 & 7.3 & 18229 & 10.2 & 30.83 & 52.3 & 22.06 & 0.2206 & 10.45 & 80.02 & \\
\hline
\end{tabular}


كركجي: دراسة مختبريه لتحسين أداء المطافح المدرجة باستخدام العوائق فوقها

\begin{tabular}{|c|c|c|c|c|c|c|c|c|c|c|c|}
\hline & 5 & 7.3 & 18229 & 10.4 & 30.83 & 52.3 & 21.64 & 0.2142 & 10.64 & 79.66 & \\
\hline & 6 & 7.3 & 18229 & 10.5 & 30.83 & 52.3 & 21.43 & 0.2112 & 10.73 & 79.48 & \\
\hline & 7 & 7.3 & 18229 & 10.8 & 30.83 & 52.3 & 20.84 & 0.2024 & 11.02 & 78.93 & \\
\hline & 8 & 7.3 & 18229 & 11.3 & 30.83 & 52.3 & 19.92 & 0.1892 & 11.50 & 78.01 & \\
\hline & 9 & 7.3 & 18229 & 11.5 & 30.83 & 52.3 & 19.57 & 0.1842 & 11.70 & 77.64 & \\
\hline & 10 & 7.3 & 18229 & 10.9 & 30.83 & 52.3 & 20.65 & 0.1997 & 11.12 & 78.74 & \\
\hline & 11 & 5.7 & 18229 & 10.8 & 39.48 & 50.7 & 20.84 & 0.2024 & 11.02 & 78.26 & \\
\hline & 12 & 5.8 & 18229 & 10.6 & 38.80 & 50.8 & 21.23 & 0.2082 & 10.83 & 78.68 & \\
\hline \multirow[t]{12}{*}{5} & 1 & 7 & 26219 & 11.55 & 46.24 & 52 & 28.03 & 0.2633 & 11.95 & 77.02 & \\
\hline & 2 & 8.2 & 26219 & 11.3 & 39.47 & 53.2 & 28.65 & 0.2721 & 11.72 & 77.97 & \\
\hline & 3 & 8.2 & 26219 & 11.65 & 39.47 & 53.2 & 27.78 & 0.2599 & 12.04 & 77.36 & \\
\hline & 4 & 8.2 & 26219 & 11.5 & 39.47 & 53.2 & 28.15 & 0.2650 & 11.90 & 77.62 & \\
\hline & 5 & 8.2 & 26219 & 11.3 & 39.47 & 53.2 & 28.65 & 0.2721 & 11.72 & 77.97 & \\
\hline & 6 & 8.2 & 26219 & 11.6 & 39.47 & 53.2 & 27.90 & 0.2616 & 12.00 & 77.45 & \\
\hline & 7 & 8.2 & 26219 & 11.6 & 39.47 & 53.2 & 27.90 & 0.2616 & 12.00 & 77.45 & \\
\hline & 8 & 8.2 & 26219 & 11.5 & 39.47 & 53.2 & 28.15 & 0.2650 & 11.90 & 77.62 & \\
\hline & 9 & 8.2 & 26219 & 11.6 & 39.47 & 53.2 & 27.90 & 0.2616 & 12.00 & 77.45 & \\
\hline & 10 & 8.2 & 26219 & 11.6 & 39.47 & 53.2 & 27.90 & 0.2616 & 12.00 & 77.45 & \\
\hline & 11 & 7 & 26219 & 11.6 & 46.24 & 52 & 27.90 & 0.2616 & 12.00 & 76.93 & \\
\hline & 12 & 7 & 26219 & 11.6 & 46.24 & 52 & 27.90 & 0.2616 & 12.00 & 76.93 & \\
\hline \multirow[t]{12}{*}{6} & 1 & 7.8 & 27221 & 12.1 & 43.08 & 52.8 & 27.77 & 0.2549 & 12.49 & 76.34 & \\
\hline & 2 & 8.8 & 27221 & 11.9 & 38.19 & 53.8 & 28.24 & 0.2614 & 12.31 & 77.13 & \\
\hline & 3 & 8.8 & 27221 & 12 & 38.19 & 53.8 & 28.01 & 0.2581 & 12.40 & 76.95 & \\
\hline & 4 & 8.8 & 27221 & 11.9 & 38.19 & 53.8 & 28.24 & 0.2614 & 12.31 & 77.13 & L \\
\hline & 5 & 8.8 & 27221 & 11.8 & 38.19 & 53.8 & 28.48 & 0.2647 & 12.21 & 77.30 & 8 \\
\hline & 6 & 8.8 & 27221 & 11.9 & 38.19 & 53.8 & 28.24 & 0.2614 & 12.31 & 77.13 & \\
\hline & 7 & 8.8 & 27221 & 12.3 & 38.19 & 53.8 & 27.32 & 0.2487 & 12.68 & 76.43 & \\
\hline & 8 & 8.8 & 27221 & 12.1 & 38.19 & 53.8 & 27.77 & 0.2549 & 12.49 & 76.78 & \\
\hline & 9 & 8.8 & 27221 & 12.55 & 38.19 & 53.8 & 26.78 & 0.2413 & 12.92 & 75.99 & \\
\hline & 10 & 8.8 & 27221 & 12.25 & 38.19 & 53.8 & 27.43 & 0.2503 & 12.63 & 76.52 & \\
\hline & 11 & 7.8 & 27221 & 12.1 & 43.08 & 52.8 & 27.77 & 0.2549 & 12.49 & 76.34 & \\
\hline & 12 & 7.8 & 27221 & 11.95 & 43.08 & 52.8 & 28.12 & 0.2597 & 12.35 & 76.60 & \\
\hline \multirow[t]{13}{*}{7} & 1 & 9.5 & 38183 & 13.55 & 49.62 & 54.5 & 34.79 & 0.3017 & 14.17 & 74.01 & \\
\hline & 2 & 10.3 & 38183 & 13.85 & 45.77 & 55.3 & 34.04 & 0.2920 & 14.44 & 73.89 & \\
\hline & 3 & 10.3 & 38183 & 13.45 & 45.77 & 55.3 & 35.05 & 0.3051 & 14.08 & 74.55 & \\
\hline & 4 & 10.3 & 38183 & 13.55 & 45.77 & 55.3 & 34.79 & 0.3017 & 14.17 & 74.38 & \\
\hline & 5 & 10.3 & 38183 & 13.6 & 45.77 & 55.3 & 34.66 & 0.3001 & 14.21 & 74.30 & \\
\hline & 6 & 10.3 & 38183 & 13.65 & 45.77 & 55.3 & 34.53 & 0.2984 & 14.26 & 74.22 & \\
\hline & 7 & 10.3 & 38183 & 13.7 & 45.77 & 55.3 & 34.41 & 0.2968 & 14.30 & 74.13 & \\
\hline & 8 & 10.3 & 38183 & 13.8 & 45.77 & 55.3 & 34.16 & 0.2936 & 14.39 & 73.97 & \\
\hline & 9 & 10.3 & 38183 & 13.9 & 45.77 & 55.3 & 33.91 & 0.2904 & 14.49 & 73.80 & \\
\hline & 10 & 10.3 & 38183 & 13.95 & 45.77 & 55.3 & 33.79 & 0.2889 & 14.53 & 73.72 & \\
\hline & 11 & 9.5 & 38183 & 13.8 & 49.62 & 54.5 & 34.16 & 0.2936 & 14.39 & 73.59 & \\
\hline & 12 & 9.65 & 38183 & 13.7 & 48.85 & 54.65 & 34.41 & 0.2968 & 14.30 & 73.83 & \\
\hline & & & & & & & & & & & \\
\hline
\end{tabular}




\section{تعليل ومناقشة النتــــائج:}

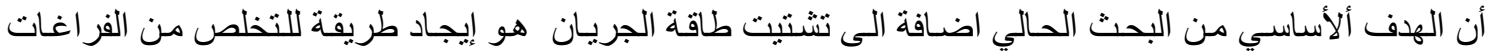

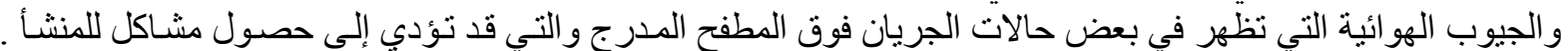

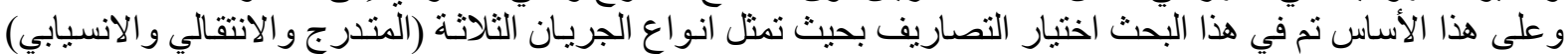

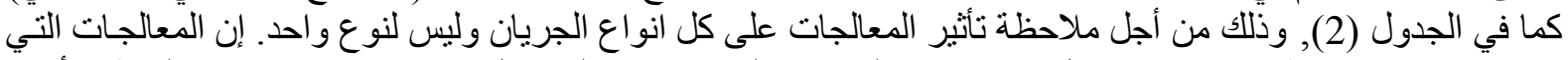

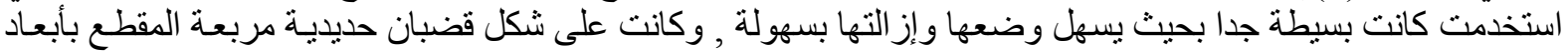

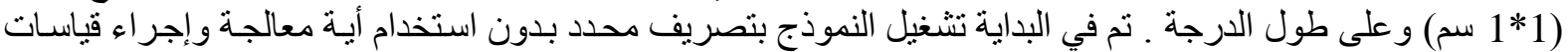

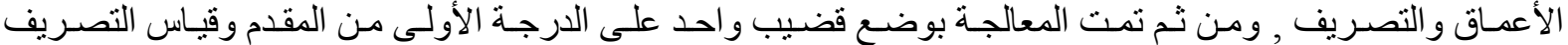

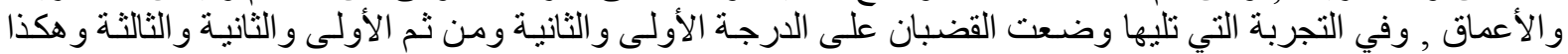

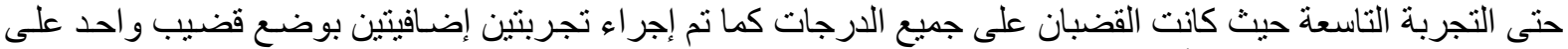

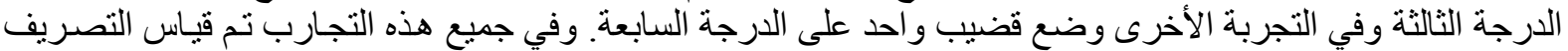

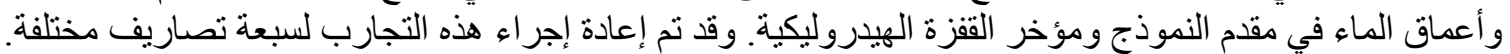

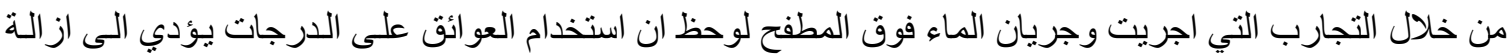

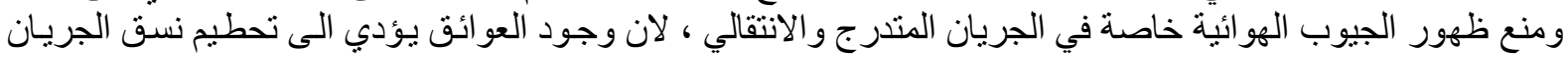

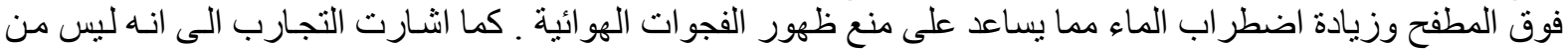

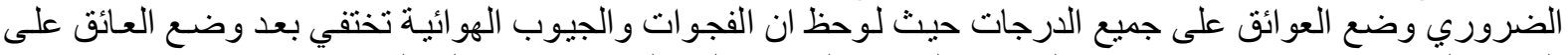

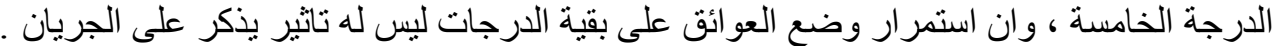

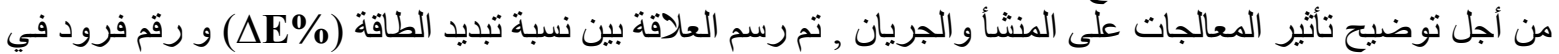

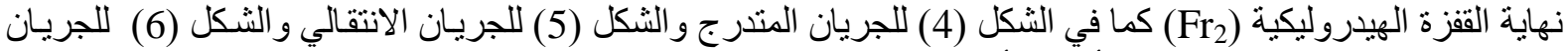

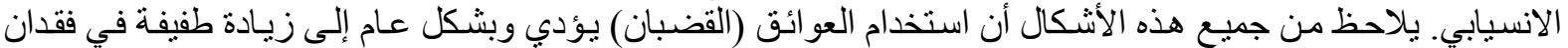

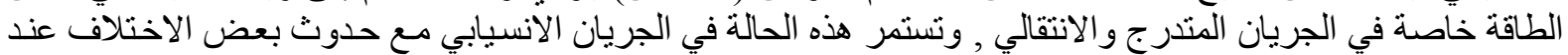

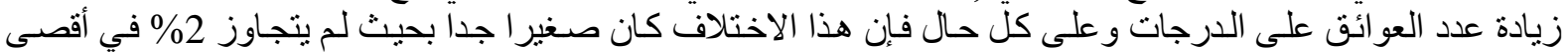

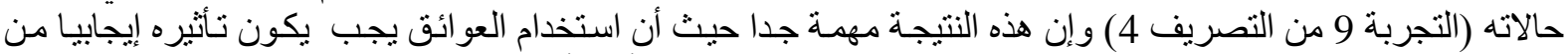

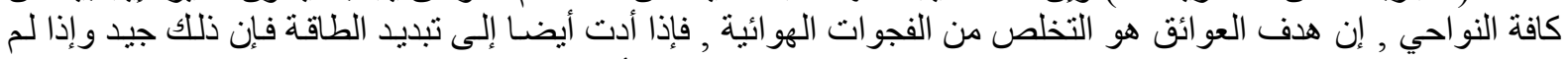

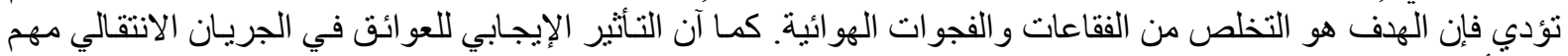
جدا لأن مشكلة الجيوب الهو ائية تظهر غالبا في هذا النوع من الجريان.

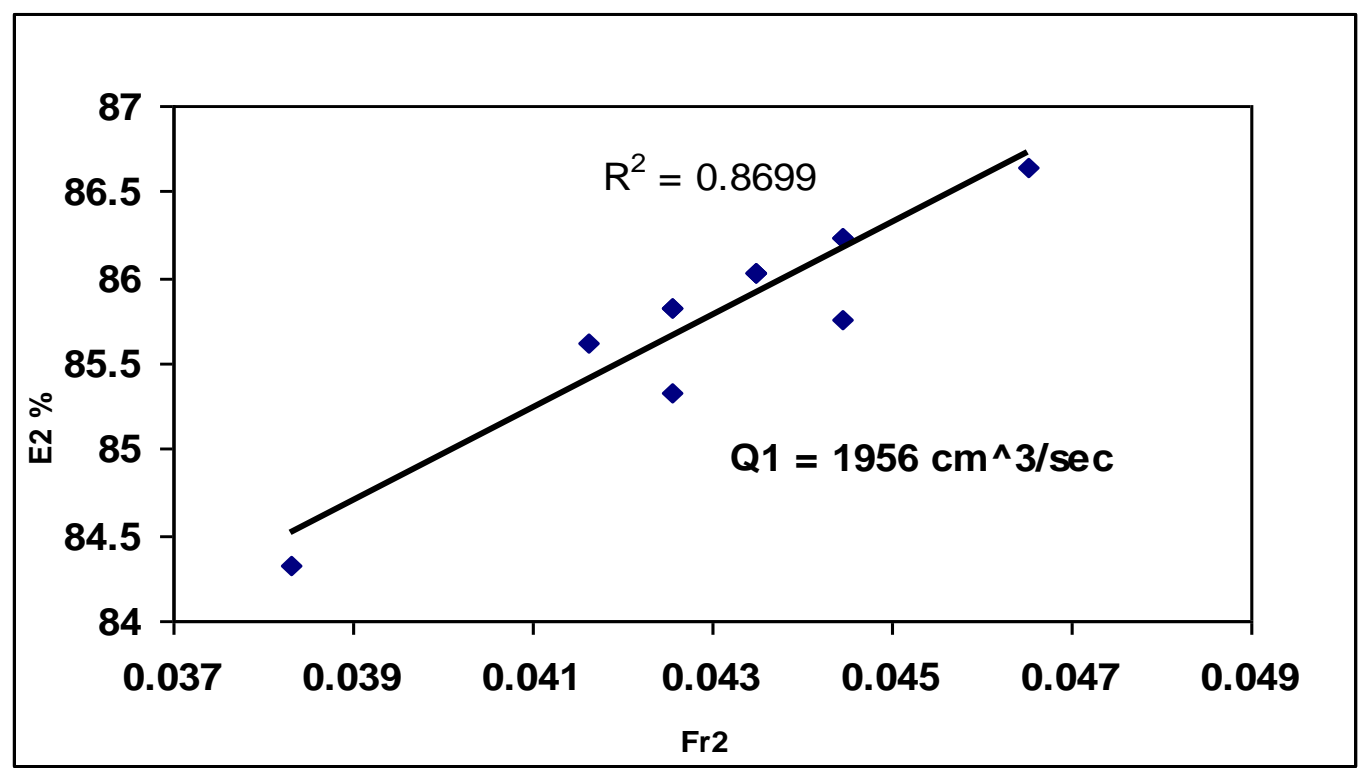

شكل (4): العلاقة بين نسبة تبديد الطاقة ورقم فرود بعد القفزة الهيدروليكية للتصريف الاول (الجريان المتدرج) 
كركجي: دراسة مختبريه لتحسين أداء المطافح المدرجة باستخدام العوائق فوقها

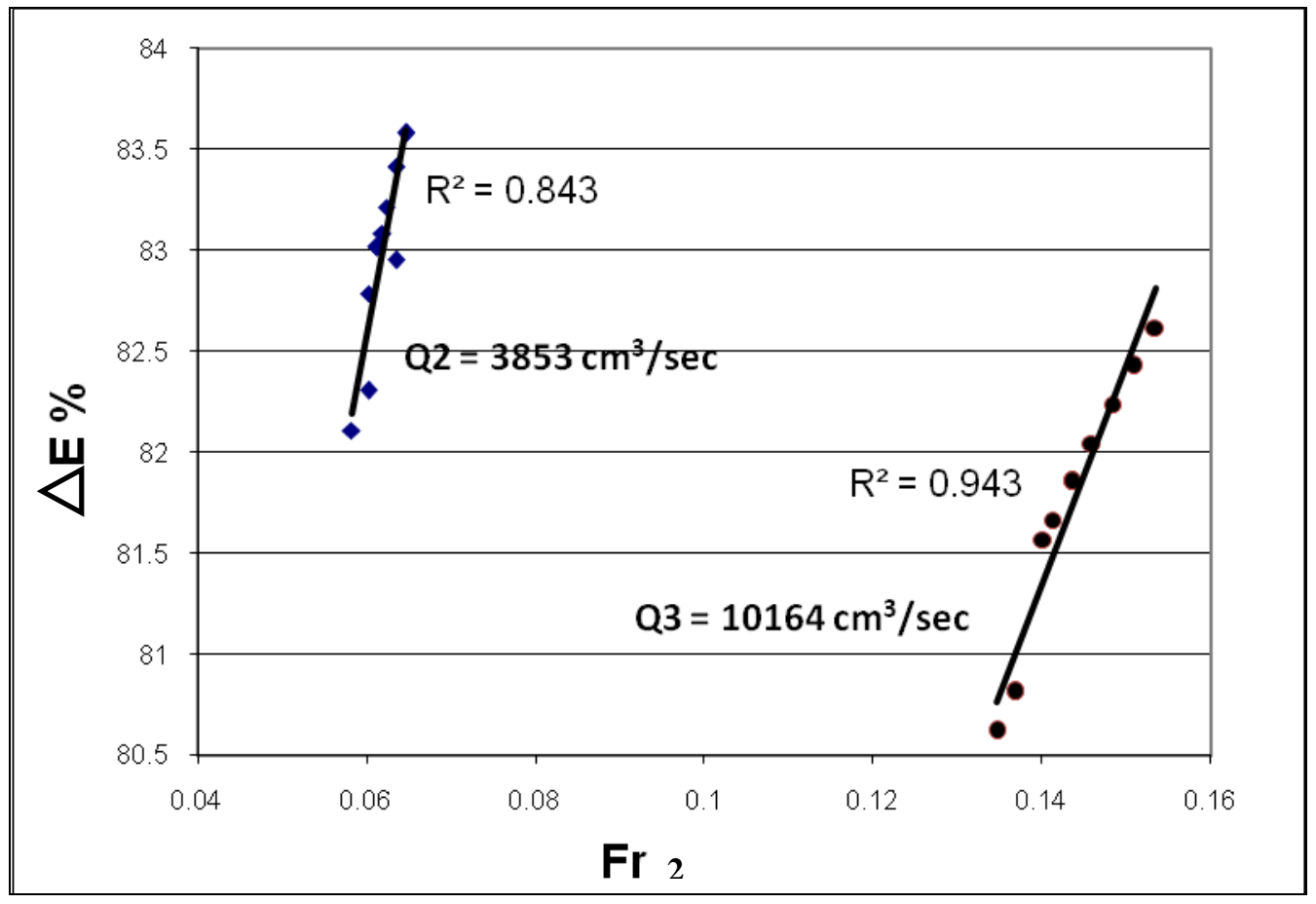

شكل (5): العلاقة بين نسبة تبديد الطاقة ورقم فرود بعد القفزة الهيدروليكية للتصاريف

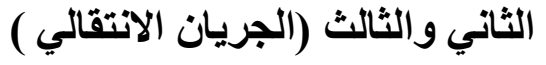

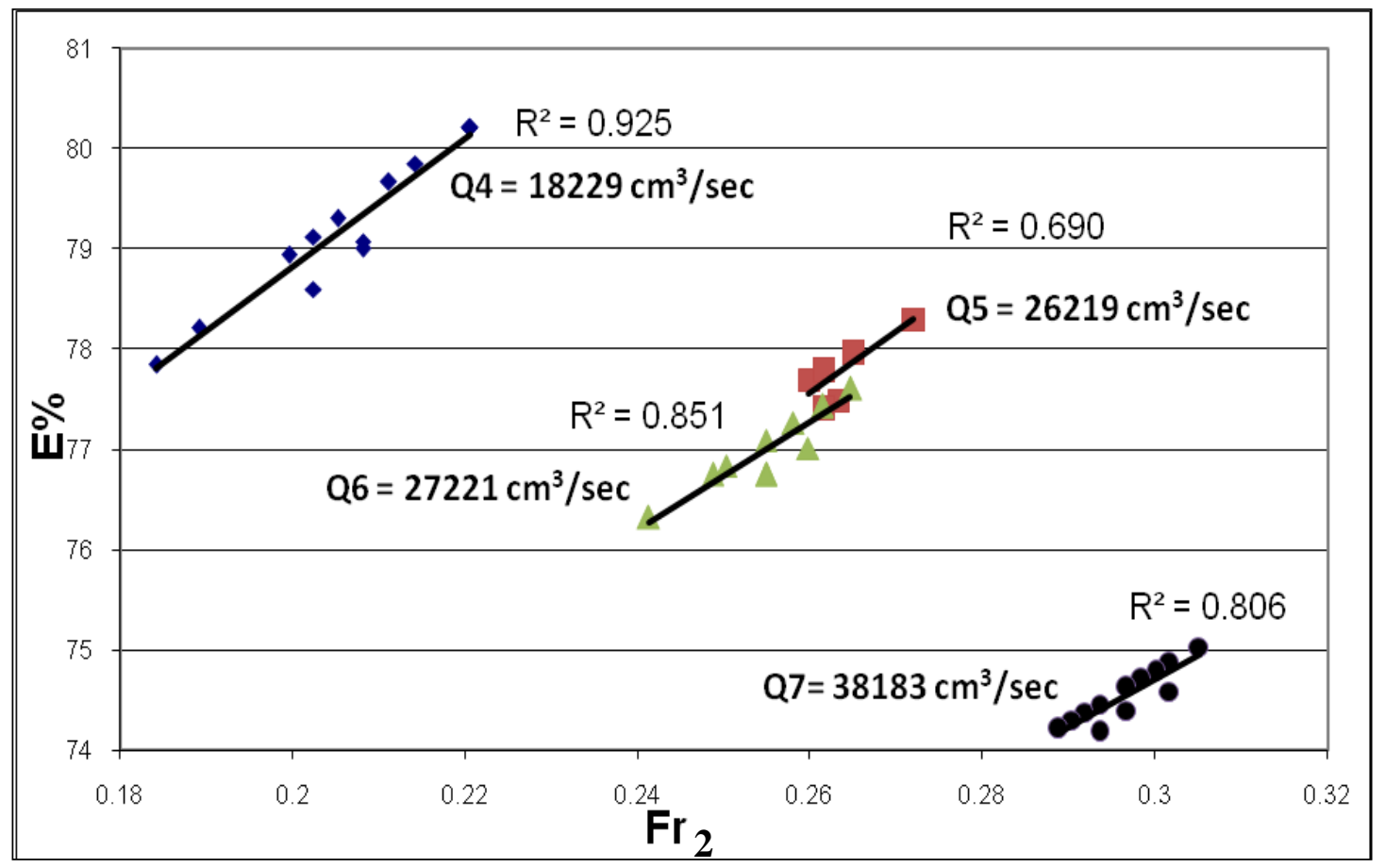

شكل (6): العلاقة بين نسبة تبديد الطاقة ورقم فرود بعد القفزة الهيروليكية

للتصاريف الرابع والخامس والسادس والسابع ( للجريان الانسيابي ) 


\section{التأثير الهياروليكي للعوائق :}

عند إجراء أي تغيير أو إضافة على منشأ هيدروليكي يجب تتبع وملاحظة تأثبر اته السلبية أو الإيجابية على الجريـان

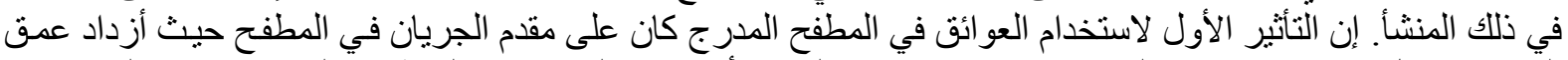

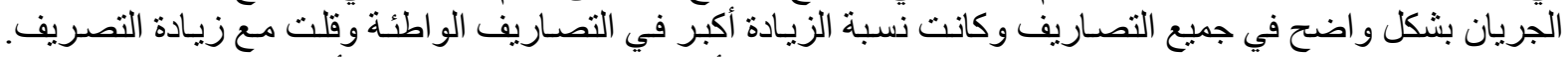
الجدول (2) يثير إلى ذلك حيث ازداد عمق الماء في التصريف الأول من 2.2سم في التجربة الأولى بدون عو التئق التق إلى 3.7

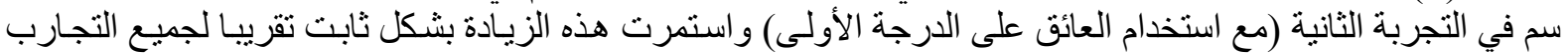

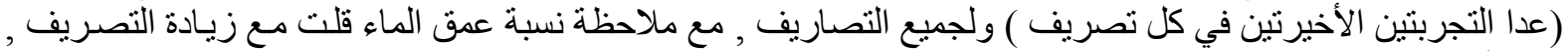
حيث أزداد عمق الماء في التصريف السابع من 9.5 سم (بدون عو ائق) إلى 10.3 سم مع استخدام العوائق ـ

أن التأثير أعلاه تلاشى عند استخدام العوائق على الدرجة الثالثة أو السابعة (التجربة الـ 11 و 12 من كل تصريف)

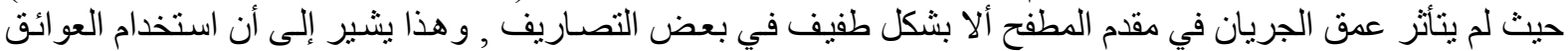

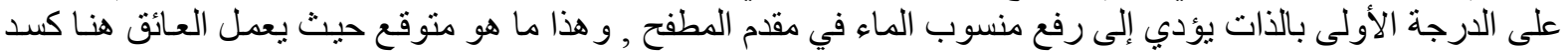

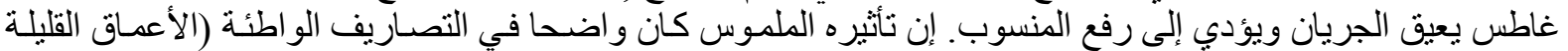

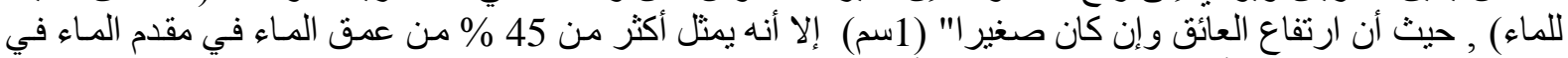

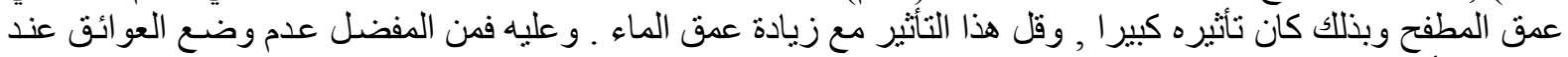

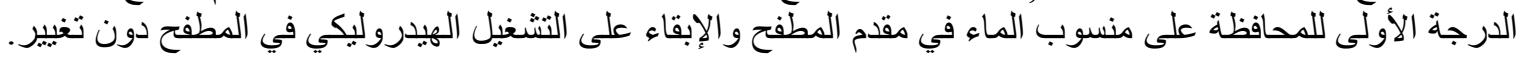

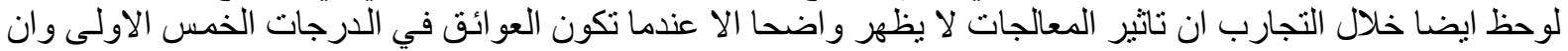

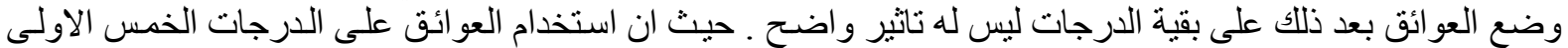

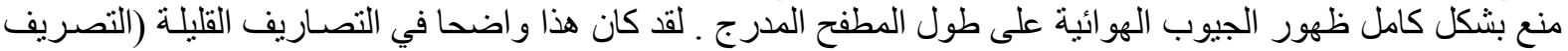

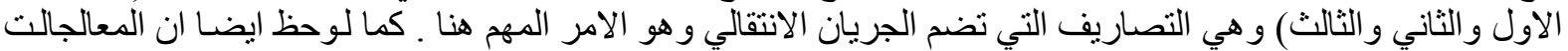

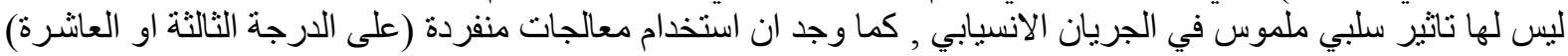

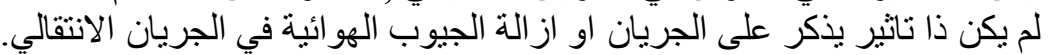

الاستنتاجات والتوصيات :

من خلال البحث والتجارب التي اجريت فيه و النتائج التي افرزتها تم التوصل الى الاستنتاجات و التوصيات التالية وضمن

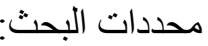

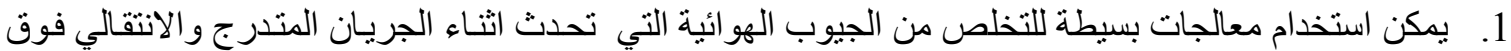

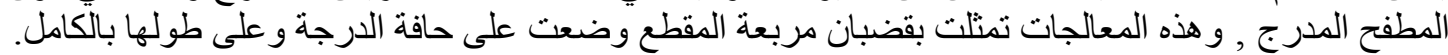

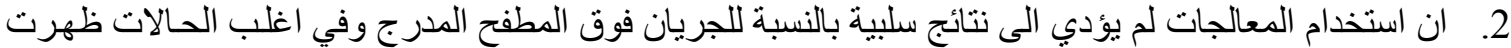
زيادة طفيفة في تثتنيت الطاقة.

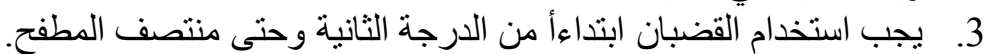

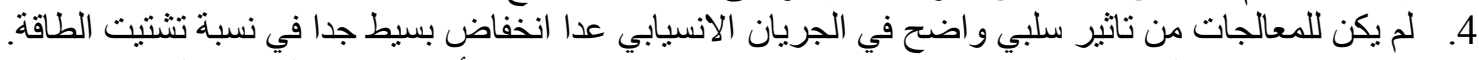

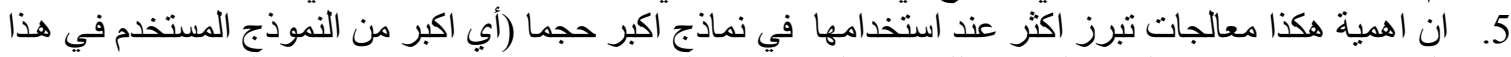

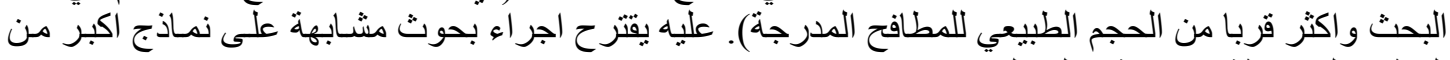
المطافح لملاحظة مدى تاثير المعالجات المني المطات

المصــادر

Ahman, M., and Zapel, E., (2000), "Stepped Spill-ways, a dissolved gas abatment alternative", In H. Minor, and W. Hager(Ed), Proceedings of the International Workshop on Hydraulics of Stepped Spillways (pp. 45-52), Zurich, Switzerland: Balkema.

Al-Ta'I , M. , and Abdulla, (2010), "Hydraulic Model Investigation of Different Configurations of Stepped Spillway". M.Sc. Dissertation, Department of Water Resources Engineering, College of Engineering, University of Baghdad, Baghdad Iraq. 
Al-Talib, A., Nasiralla, (2007), "Laboratory Study of Flow Energy Dissipation Using Stepped Weirs", M.Sc. Dissertation, Department of Water Resources Engineering, College of Engineering, University of Mosul, Mosul- Iraq.

Barani, G., Rahnama, M., and Sohrabipoor, N. (2005), "Investigation on Flow Energy Dissipation over Differnet Stepped Spillways", American Journal of Applied Sciences, 2(6), 1101-1105.

Chanson, H., (1993), "Stepped Spillways Flow Air Entrainment", Canadian Journal of Civil Engineering, 20(3), 422-433.

Chanson, H., (1994), "Comparison of Energy Dissipation Between Nappe and Skimming Flow Regimes on Stepped Chutes", Journal of Hudraulic Research, IAHR, 32 (2), 213-218.

Chow, V.T., (1959), "Open- Channel Hydraulics", NewYork: McGraw-Hill.

Christodoulou, G., (1995), "Energy Dissipation on Stepped Spillways Closure", Journal of Hydraulic Engineering, ASCE. 121 (1), 85-87.

Peruginelli, A., and Pagliara, S., (2000), "Energy Dissipation Comparison Among Stepped Channel, Drop and Ramp Structures", In H. Minor, and W. Hager (Ed.), Proceedings of International Workshop on Hydraulics of Stepped Spillways. Zurich, Switzerland. Balkema.

Peyras, L. Royet, P., and Degoutte, G., (1992), "Flow and Energy Dissipation over Stepped Gabion Weirs", Journal of Hydraulic Engineering, ASCE, 118, 707-717.

Sorenson, R., (1985), "Stepped Spillway Hydraulic Model Investigation", Journal of Hydraulic Engineering, ASCE, 111, 1461-1472.

Yousif, E., Easa, (2006), "Laboratory study of Aggradation Characteristics Upstream Dams", M.Sc. thesis, Department of Water Resources Eng., College of Eng., University of Mosul, Mosul-Iraq.

\section{قائمة الرموز المستخذمة}

\begin{tabular}{|c|c|c|}
\hline الوحدة & معنى الرمز & الرمز \\
\hline سم & عرض القناة & $\mathrm{B}$ \\
\hline سم & طاقة الجريان الكلية مقدم المطفح & $\mathrm{E}_{\mathrm{t}}$ \\
\hline سم & طاقة الجريان الكلية مؤخر القفزة الهيدروليكية & $\mathrm{E}_{2}$ \\
\hline- & رقم فرود مؤخر الققزة الهيدروليكية & $\mathrm{Fr}_{2}$ \\
\hline سم/ثانية2 & التعجيل الأرضي & $\mathrm{G}$ \\
\hline سم & ارتفاع الماء فوق الهدار القياسي & $\mathrm{H}$ \\
\hline سم & ارتفاع المطفح & $\mathrm{P}$ \\
\hline سم ${ }^{3}$ /ثانية & التصريف & Q \\
\hline سم/ثانية & سر عة الجريان مقدم المطفح & $\mathrm{V}_{\mathrm{o}}$ \\
\hline سم/ثانية & سر عة الجريان بعد الققزة الهيدروليكية & $\mathrm{V}_{2}$ \\
\hline سم & ارتفاع الماء فوق مقدمة المطفح & $\mathrm{y}_{\mathrm{o}}$ \\
\hline سم & ارتفاع الماء بعد الققزة الهيدروليكية & $\mathrm{y}_{2}$ \\
\hline
\end{tabular}

$$
\text { تم اجراء البحث في كلية ألهندسة = جامعة ألموصل }
$$

